\title{
Positive affect and mindfulness as predictors of resilience amongst women leaders in higher education institutions
}

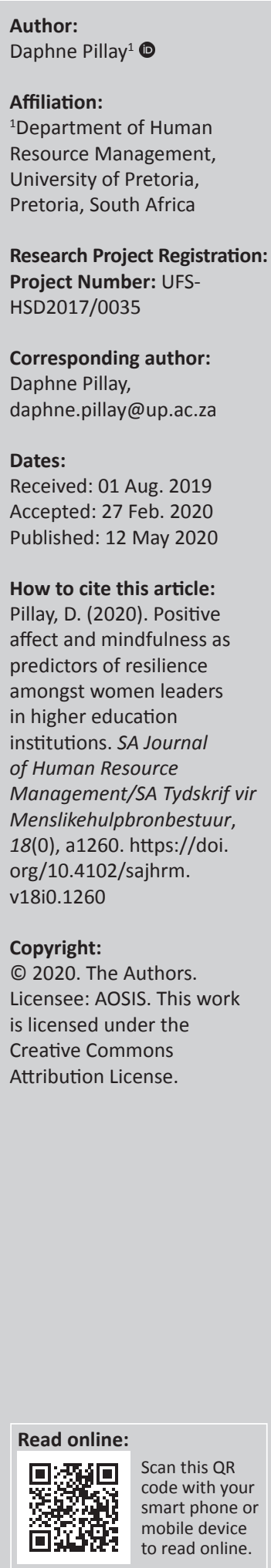

Orientation: Psychological resources are the factors that appear to have a significant impact on how leaders adapt to adversity and remain resilient. Positive affect and mindfulness are the psychological resources that positively relate to the levels of resilience of women leaders in higher education institutions.

Research purpose: The purpose of this study was to investigate the effect of positive affect and mindfulness on the levels of resilience of women leaders in higher education institutions in South Africa.

Motivation for the study: Identifying the internal resources women leaders use to facilitate resilience will allow higher education institutions to prioritise these resources in leadership support programmes to assist these women leaders.

Research approach/design and method: A cross-sectional survey design was used to collect data in a purposive sample of $n=255$ women leaders in four South African higher education institutions. Pearson's correlation analyses, multiple regression analyses and mediation analysis were used to analyse the data.

Main findings: Findings indicated that positive affect and mindfulness were found to be significant predictors of resilience. Additionally, mindfulness was found to be a partial mediator in the relationship between positive affect and resilience.

Practical/managerial implications: Higher education institutions can assist women leaders by investing in psychological resources such as mindfulness and positive affect to enhance the levels of resilience.

Contribution/value-add: This study contributes to the limited research on the role of internal resources to enhance resilience in a workplace setting and more specifically amongst women leaders.

Keywords: resilience; positive affect; mindfulness; women leaders; higher education.

\section{Introduction}

Historical events in South African society have given rise to a cycle of underrepresentation of women in leadership roles in tertiary institutions in South Africa. However, higher education institutions are making steady advances towards transformation, which is indicated by the increase of women leaders in positions of leadership. According to Sanaghan (2016), the volatile environment that characterises the South African higher education system presents a barrier to leadership growth. Howe-Walsh and Turnbull (2016) state that these barriers are persistent and require women leaders to continuously use resilience as a key resource to resume optimal functioning following the experience of a setback. To this end, the concept of resilience serves as a crucial resource for women leaders and, as a result, higher education institutions need to prioritise resilience development for these women to adapt to the uncertainty (Sanaghan, 2016). Weatherspoon-Robinson (2013) asserts that for women leaders to remain persistent and overcome these barriers, they need to possess the resources to resist disruptions that threaten their career journey especially in an environment that is less than optimal. This includes the capacity to rebound from setbacks and disruptions as well as to deal successfully with less than adequate employment environments while being aware that these inadequacies exist. Authors in the field of leadership development (Foerster \& Duchek, 2018) state that traits such as sense of control and 
optimism needed to promote persistency during challenging situations are indicative of resilience. According to Ledesma (2014), resilience is a crucial resource for leaders as it contributes to their survival, adaptation and success, especially in a complex working environment. In a review of top women executives in higher education, WeatherspoonRobinson (2013) argues that a key trait of successful leaders is not how they thrive during optimal working conditions, but rather how they exhibit psychological strength, perseverance and optimism during demanding circumstances. As a result, resilience can serve as a resource for coping with complex situations, which is especially crucial for women leaders in higher education.

In investigating the processes that explain resilience, there are a growing number of authors (Lee, Cheung, \& Kwong, 2012; Manomenidis, Panagopoulou, \& Montgomery, 2019) who have been promoting the role of internal resources as being influential in the resilience process. Internal resources can be defined as positive attributes of the individual that assist the process of development, adaptation and resilience (Mould, 2014). There have been several scholars who insist that despite decades of profound advances in research on the multidimensional concepts of resilience, the urgency and need for programmes to promote personal and internal resiliency factors amongst individuals have never been more pronounced (Lee et al., 2012; Mould, 2014; Kinman \& Grant, 2017). According to Masten (2011), there is an urgent need for researchers to understand the strengths and positive adaptations that are required to decrease the consequences of persistent barriers and obstacles. As a result, the role of protective processes that are embedded within the individual, which include traits, character strengths and internal resources, must be emphasised. Lee et al. (2012) reported that in a meta-analysis that investigated the factors explaining resilience amongst individuals, factors such as self-efficacy, optimism, social support, self-esteem and life satisfaction had a stronger association with resilience compared with risk factors. This suggests that attempts made to increase individual levels of resilience would be more effective if internal resiliencerelated factors are highlighted as opposed to focusing on risk factors (Lee et al., 2012). By using this as a point of departure, this article adopts an individual-level perspective in investigating the internal resources used by women leaders in higher education.

Ong, Kim, Young, and Steptoe (2017) define positive affect as a state of pleasurable engagement with the environment that elicits feelings, such as happiness, enjoyment, passion and contentment that includes both enduring moods (e.g. affective traits) and short-term emotions (e.g. dynamic states). In addition to the positive affect, researchers have also explored the concept of negative affect, which reflects a general state of anguish and unpleasurable engagement comprising a variety of negative mood states including rage, hatred, disgust, guilt, dread and anxiety. For the purpose of this study, only positive affect will be considered as the focus is on resources that can enhance resilience, although research (Aydemir, 2018; Tugade \& Fredrickson, 2004) has indicated that negative affect significantly decreases the levels of resilience. Research conducted (Tugade \& Fredrickson, 2004) in resilience has consistently reported that resilient individuals display a positive, hopeful and spirited attitude to life and life's challenges, which is also referred to as positive affect. According to Werner and Smith (1992), this is primarily because resilient leaders are aware that remaining positive increases the possibility of overcoming challenging circumstances. As a result, they strive to enhance their positive emotionality through hopeful and optimistic thinking. Tugade and Fredrickson (2004) agree, arguing that positive mood states promote sustained resilience in the face of adversity, because an optimistic view of life makes one better able to handle stressors and challenges.

Similar to positive affect, there is a growing body of research (Halliday, 2018; Keye \& Pidgeon, 2013) which suggests that mindful individuals tend to display an increased propensity to be resilient in the face of challenges. Brown and Ryan (2003) define mindfulness as a psychological state that encompasses being attentive to and aware of what is taking place in the present. According to Bartz (2017), a key attribute of resilience is being able to adapt to adversity based on the characteristics of the situation. By paying attention to and being aware of incoming stimuli, the mindful individual is able to respond in an adaptive manner, which increases their ability to become resilient.

Despite the growing body of evidence (Bartz, 2017; Tugade \& Fredrickson, 2004), which advocates for the role of positive affect and mindfulness in influencing resilience, there is still a growing need for studies that attempt to investigate how these variables work to influence resilience in the work context and more specifically amongst women leaders. Several studies focus on the role of positive affect and mindfulness in influencing resilience in clinical samples, with emphasis on increasing resilience for dealing with clinical issues such as health-related problems (Arewasikporn, Turner, Alschuler, Ehde, \& Jensen, 2018; Collins, Stritzke, Page, Brown, \& Wylde, 2018; Kong et al., 2018). There are a limited number of studies that attempt to determine how positive affect and mindfulness influence resilience to workrelated challenges and more specifically amongst women leaders (Rupprecht et al., 2019; Sommer, Howell, \& Hadley, 2016). This study makes a significant contribution to the field of positive psychology at work as it sheds new light on the usefulness of combining both positive affect and mindfulness to influence resilient outcomes and adds new knowledge to this developing field.

\section{Purpose}

The purpose of this study is exploratory in nature and is aimed at investigating the effect of positive affect and mindfulness on resilience amongst women leaders in higher 
education institutions. The role of mindfulness in influencing the relationship between positive affect and resilience was investigated in this regard. The study aims to test the assumption made by early cognitive-emotive theories, which maintain that affect has a substantial influence on cognitive processes, particularly attention and awareness and, as a result, serves to regulate the selectivity of attention as well as motivating action and behaviour (Tyng, Hafeez, Mohamad, Saad, \& Malik, 2017). By viewing mindfulness as a cognitive process that may be influenced by emotional processes, that is, positive affect, the current study aims to determine if the combination of these two variables can enhance the overall effect on resilience.

\section{Literature review}

\section{Resilience, positive affect and mindfulness}

According to Hamby, Banyard, and Grych (2016), resilience consists of a process of effectively coping with and adapting to adverse circumstances, threat, loss and stressful situations. Other authors (Grover, Teo, Pick, Roche, \& Newton, 2018; Petrosyants, 2017) define resilience as a psychological strength used to deal with challenging circumstances. Richardson, Neiger, Jensen, and Kumpfer (1990) state that when used as a resource, resilience allows an individual to resume the prior state of functioning after being exposed to demanding life conditions, threat and difficulty. The authors go on to add that in certain instances resilience may even allow the individual to function at an elevated level compared with functioning before the adverse circumstance. Despite the various definitions offered by different authors, there are key commonalities that are consistent in the understanding of resilience in positive psychology. Firstly, several authors (Fergus \& Zimmerman, 2005; Oshri, 2019; Tugade \& Fredrickson, 2004) agree that resilience is developmental in nature and is manifested through effective adjustment, rebounding back from setbacks and cultivating psychological resources to overcome adversity. Secondly, resilience should be understood as the outcome that occurs through a developmental process, which is manifested through competence to deal with challenging situations (Chmitorz et al., 2018; Ungar, 2004). Finally, a resilient response is heightened during demanding situations such as exposure to pressures, strain, obstacles and trials (Dannels, \& Masters, 2020; Howard \& Johnson, 2002).

This study will adopt a micro-level approach in investigating resilience by understating the individual resources that can assist women leaders in increasing their levels of resilience. Positive affect has been identified as an internal strength that women leaders may draw upon when facing workplace challenges and obstacles. According to Fredrickson (2004), positive affect increases the propensity to experience positive emotions such as joy, interest, contentment and love. This heightened presence of positive mood states leads to the alleviation of negative emotions such as worry, unhappiness and rage. Tugade and Fredrickson (2004) maintain that positive emotions as a by-product of positive affect serve as a buffer against stressors and therefore increase optimal functioning. According to Van Cappellen, Toth-Gauthier, Saraglou, and Fredrickson (2016), positive emotions work by widening one's transient thought-action repertoire. The broadened thought-action repertoire allows for the use of constructive coping strategies, such as positive reappraisal, problem-focused coping and allocating positive significance to everyday events (Folkman \& Moskowitz, 2000). This suggests that positive affect is a valuable tool for enhancing resilience as it gives rise to positive emotions, which promote adaptive responses to stress and adversity (Tugade \& Fredrickson, 2004). By minimising negative emotions, positive emotions promote resilience because the absence of threats means that the individual is free to engage in exploratory behaviours (Tugade \& Fredrickson, 2004). This is expected to develop personal capacities or resources, including coping resources. As a result, positive affect enhances crucial personal strengths such as resilience to negative circumstances.

In addition to exploring the role of positive affect in increasing the levels of resilience for women leaders, this study will also explore the impact of mindfulness on resilience. Mindfulness refers to the ability to actively regulate one's attention towards the present, in a transparent and accepting manner (Brown \& Ryan, 2003). Halliday (2018) asserts that by emphasising attention on present experiences, mindfulness has the ability to facilitate adaptive coping to demanding situations. According to Bishop et al. (2004), mindfulness allows the individual to abandon any effort to control cognitions and instead emphasises that those cognitions and feelings are indeed present. The current study regards mindfulness as a psychological trait that enables one to attend to and be cognisant of experiences in the present moment (Brown \& Ryan, 2003). This allows one to curb involuntary cognitions and maladaptive behavioural responses, and engage in the promotion of informed, selfapproved behavioural regulation, which is linked to an enhanced state of well-being (Ryan \& Deci, 2000). Keng, Smoski, and Robins (2011) maintain that mindfulness draws attention to the momentary nature of destructive cognitions, feelings and physical sensations, allowing the adoption of a flexible and impartial response rather than one that is exaggerated. This cognitive pattern is known to foster adaptive functioning and allow for reintegration after adversity. Being mindful of present experiences allows one to recognise destructive emotions and to be fully aware of the impact such emotions can have on one's response to challenges. In addition to exploring the direct effect of mindfulness on resilience, the current study investigates the extent to which mindfulness influences resilience through interacting with positive affect.

\section{The relationship between positive affect, mindfulness and resilience}

Tugade and Fredrickson (2004) argue that a positive appraisal style positively influences one's well-being, by increasing the likelihood of the individual being resilient. 
Results of a study conducted by Martínez-Marti and Ruch (2017) support the claim made by Tugade and Fredrickson (2004) by reporting a strong positive correlation between positive affect and resilience. Tugade and Fredrickson (2004) state that individuals who have a positive disposition towards life are generally in a better position to deal with stressors and challenges, which makes them more resilient. Fredrickson (2001) uses the principles of the broaden-andbuild theory to explain the relationship between positive affect and resilience. According to the broaden-and-build theory, positive emotions that are produced through positive affect have exclusive and harmonising adaptive functions, which manifest in physiological effects. Positive emotions are linked to responses that broaden one's thoughts and actions, which in turn build significant personal resources that increase the likelihood of remaining resilient during adversity (Fredrickson, 2001). As a result, positive affect through the creation of positive emotions may significantly influence the levels of resilience in the current sample:

Hypothesis 1: Positive affect is a significant predictor of resilience

Keye and Pidgeon (2013) reported a strong positive relationship between mindfulness and resilience. According to the authors, mindfulness as a resource forms part of the repertoire of psychological strengths that allow individuals to successfully navigate change. Adams et al. (2016) state that mindfulness has the ability to promote self-awareness, which assists in enhancing resilience. Being self-aware allows the individual to identify the signs of stress allowing them to respond more effectively. Adams et al. (2016) state that mindfulness increases the capacity to recognise the power of thoughts and increases the chances of finding ways to skilfully work with them. This is a critical aspect needed to remain resilient, as individuals need to be able to discriminate between thoughts that nurture or deplete internal resources. This suggests that mindfulness may play a significant role in influencing resilience:

Hypothesis 2: Mindfulness is a significant predictor of resilience

Although researchers (Martínez-Marti \& Ruch, 2017; Keye \& Pidgeon, 2013; Tugade \& Fredrickson, 2004) have documented the role of positive affect and mindfulness in individually predicting resilience, there is a dearth of studies that attempt to investigate the indirect relationships between these variables. There is an emerging body of research, which alludes to the notion that emotion may play a significant role in cognitive processing (Kret \& Bocanegra, 2016; Tyng et al., 2017). According to Dane (2011), cognitive processing is an integral aspect of mindfulness because being able to pay attention to the present moment requires the individual to process both internal and external stimuli. Brosch, Scherer, Grandjean, and Sander (2013) add to this argument by stating that emotional responses (displaying positive affect) may modulate and guide cognition to enable adaptive responses to the environment. Because mindfulness is a cognitive process, this suggests that positive affect may indirectly influence resilience through influencing the cognitive process, i.e. mindfulness. This is supported by the broaden-and-build theory of positive emotions (Fredrickson, 2001), which states that proportional to neutral states and routine action, positive emotions as a by-product of positive affect widen people's transient thought-action repertoires. Thus, through the creation of positive emotion, positive affect may broaden an individual's thought processes to encourage awareness and attention. This in turn may positively influence how the individual responds to challenges during time of threat, which increases resilient outcomes:

Hypothesis 3: There is an indirect relationship between positive affect and resilience through mindfulness

\section{Research design}

\section{Research approach}

A quantitative research design was adopted to test the hypotheses proposed in the current study regarding the effect of positive affect and mindfulness on resilience. By using a cross-sectional approach to research, self-report measures were used to collect data on the biographical variables, positive affect, mindfulness and resilience.

\section{Research method}

Participants: A non-probability sampling design using purposive sampling was selected for this study. According to Huysamen (2001) in purposive sampling, the researcher uses existing knowledge about the sample to intentionally secure participants in such a manner that the participants secured are regarded as representative of the relevant population. The current study was specifically interested in women leaders in the higher education sector, and therefore a purposive sampling method was considered appropriate.

Participants who were selected for the study were drawn from both Traditional Universities and Universities of Technology in South Africa. Because the focus of the study was on identifying internal resources used by women leaders, an all-women sample consisting of women who occupied leadership positions in both the academic and support services was targeted. Through liaising with the relevant Human Resources (HR) department of each institution, a list of $n=1193$ potential participants from four institutions was identified. Unfortunately, information regarding the total number of leadership positions in each institution was not made available and, therefore, a comparison between the number of male and female leaders in each institution could not be made. Out of the $n=1193$ questionnaires distributed via an online survey system, only 255 questionnaires were returned, achieving a response rate of $21.3 \%$. In a study conducted by Nulty (2008), examining the differences in response rates for online and paper-based 
TABLE 1: Biographical details of the sample.

\begin{tabular}{|c|c|c|}
\hline Characteristic & Frequency & Percentage \\
\hline \multicolumn{3}{|l|}{ Age (years) } \\
\hline $26-34$ & 16 & 6.3 \\
\hline $35-44$ & 67 & 26.3 \\
\hline $45-54$ & 100 & 39.2 \\
\hline 55 and older & 70 & 27.5 \\
\hline \multicolumn{3}{|l|}{ Marital status } \\
\hline Single & 51 & 20.0 \\
\hline Married & 157 & 61.6 \\
\hline Divorced & 24 & 9.4 \\
\hline Widowed & 8 & 3.1 \\
\hline Other & 7 & 2.7 \\
\hline \multicolumn{3}{|c|}{ Highest educational qualification } \\
\hline Grade 12 & 1 & 0.4 \\
\hline Higher certificate/diploma & 5 & 2.0 \\
\hline Bachelor's degree & 15 & 5.9 \\
\hline Honours/master's degree & 91 & 35.7 \\
\hline PhD & 141 & 55.3 \\
\hline \multicolumn{3}{|l|}{ Category of employment } \\
\hline Academic & 142 & 55.7 \\
\hline Support services & 104 & 40.8 \\
\hline \multicolumn{3}{|l|}{ Tenure } \\
\hline Less than a year & 17 & 6.7 \\
\hline $1-2$ years & 29 & 11.4 \\
\hline $3-5$ years & 55 & 21.6 \\
\hline $6-10$ years & 59 & 23.1 \\
\hline More than 10 years & 90 & 35.3 \\
\hline
\end{tabular}

surveys, the author concluded that a typical response rate for online surveys ranges between $20 \%$ and $47 \%$. This suggests that the low response rate received in the current study is in line with other studies using online surveys. According to Hoddinott and Bass (1986), when analysing the response rates of surveys, it is not the response rate that is crucial but rather the quality of the survey or the data.

The biographical details of the sample are presented in Table 1.

\section{Measurement instruments}

A questionnaire consisting of three self-report measures was used to obtain quantitative data for the current study. Each instrument has reported a well-established reliability and validity for use with different samples. The Cronbach's alpha coefficients were used as an estimate of reliability for all instruments.

Resilience: The 14-item Resilience Scale (RS-14) as derived from the resilience scale by Wagnild and Young (1993) was used to measure resilience in the current study. The original resilience scale consisted of 25 items and was later adapted by Wagnild (2009) to form a 14-item scale (RS-14). The RS-14 is scored on a seven-point Likert-type scale with scores ranging from 1 (strongly disagree) to 7 (strongly agree). Examples of items include 'I generally take things in my stride' and 'when in a difficult situation, I can generally find my way out of it'. The RS-14 reported an alpha coefficient of 0.88 in the current study.
Positive affect: The Positive and Negative Affectivity Schedule (PANAS) (Watson, Clark, \& Tellegen, 1988) was used to measure positive affect. The PANAS is a 20-item questionnaire that is scored on a five-point Likert scale ranging from 1 (very slightly or not at all) to 5 (extremely). The PANAS consists of 10 items that measure positive affect and 10 items measuring negative affect. Participants are asked to indicate the extent to which they have felt the different emotions such as excited, active, sad and upset. High scores on the positive affect scale indicate high levels of positive affect. For the purpose of this study, only the positive affect scale was used. A reliability estimate of 0.85 was reported for the positive affect dimension in the current study.

Mindfulness: The Mindfulness Attention Awareness Scale (MAAS) by Brown and Ryan (2003) was used to examine mindfulness. The MAAS is a 15-item questionnaire scored on a six-point Likert scale. The scale ranges from 1 (almost always) to 6 (almost never). The MAAS is unidimensional in nature and attempts to measure levels of dispositional mindfulness. Examples of items from the MAAS include 'I find it challenging to remain focused on what's happening in the present' and 'I hurry through activities without being really attentive to them'. Within the current sample, the MAAS reported an alpha coefficient of 0.89 .

\section{Data collection procedure}

Following the gatekeeper permission received from the four South African universities, arrangements were made with the human resources department of each institution to identify women leaders who met the criteria set out in the current study, i.e. occupying a leadership position in the academic and support services occupational categories. An e-mail list of all possible participants was provided to the researcher and e-mail links were sent out by using the electronic EvaSys survey system. An e-mail together with the link to the questionnaire and the letters of informed consent and participant information letter was sent to all participants. The completed questionnaires were sent to a data bank created solely for the purpose of this study via the EvaSys system.

\section{Statistical analysis}

By using the Statistical Package for the Social Sciences (SPSS) version 25, descriptive and inferential statistics were conducted to investigate reliability and to analyse the data. PROCESS macro (Hayes, 2013) was used to determine the mediating effect of mindfulness on the relationship between positive affect and resilience.

\section{Ethical consideration}

Full ethical clearance was obtained from the Research Ethics Committees and Registrars of the four institutions that were selected for this study. Participants were informed about 
their rights within the context of the study as well as how the data generated from the questionnaires would be used and stored. Participants were also reminded that participation in the study was voluntary and that they were free to withdraw should they feel inclined.

\section{Results \\ Descriptive statistics and correlations}

As indicated in the correlation analysis shown in Table 2, both positive affect (0.507) and mindfulness (0.311) reported statistically significant relationships with resilience. Positive affect reported a large effect with resilience although mindfulness reported a medium effect. All correlations were statistically significant $(p<0.01)$.

The mean score reported for resilience $(\mathrm{M}=5.97 ; \mathrm{SD}=0.65)$ suggests that women leaders in the current sample display moderately high levels of resilience. This was expected, given that participants were already in leadership positions. To reach and maintain their respective leadership levels, a degree of resilience was required. Women leaders in the current sample reported high levels of positive affect $(\mathrm{M}=4.05 ; \mathrm{SD}=5.22)$ and levels of mindfulness were moderately high $(\mathrm{M}=4.27 ; \mathrm{SD}=0.80)$. All instruments reported more than acceptable reliability estimates.

\section{Multiple regression analysis}

Multiple regression analysis was conducted to test hypotheses 1 and 2 proposed in this study. In the analysis, resilience was used as the dependant variable, with positive affect and mindfulness being used as the independent variables. As indicated in Table 3, the model was statistically significant $(F=50,59 ; p<0.001)$ with positive affect and mindfulness explaining $28 \%$ of the variance in resilience. Although both positive affect $(\beta=0,46 ; t=8,19 ; p<0.000)$ and mindfulness $(\beta=0,18 ; t=3,22 ; p<0.001)$ are significant predictors of resilience, from the beta coefficient it becomes clear that positive affect explains a greater proportion of variance in resilience.

TABLE 2: Descriptive statistics and correlations amongst the variables.

\begin{tabular}{lcccccc}
\hline Variable & $\mathbf{M}$ & SD & $\boldsymbol{\alpha}$ & $\mathbf{1}$ & $\mathbf{2}$ & $\mathbf{3}$ \\
\hline 1. Resilience & 5.97 & 0.65 & 0.88 & - & - & - \\
2. Positive affect & 4.05 & 0.52 & 0.85 & $0.507 *$ & - & - \\
3. Mindfulness & 4.27 & 0.80 & 0.89 & $0.311^{*}$ & $0.289 *$ & - \\
\hline
\end{tabular}

$\mathrm{M}$, mean; SD, standard deviation; $\alpha$, Cronbach's alpha.

$*, p \leq 0.01$.

\section{Mediation analysis}

Each component of the proposed mediation model was assessed using mediation analysis (Hayes, 2013). The independent variable in the analysis was positive affect $(X)$; the dependant variable was resilience $(\mathrm{Y})$ and the mediator was mindfulness $(\mathrm{M})$. The results of the mediation analysis suggested that positive affect significantly influences resilience $(\beta=0.79, t=8.19, p<0.001)$. It was also found that positive affect significantly predicts mindfulness $(\beta=0.67$, $t=4.80, p<0.001)$. Finally, it was found that mindfulness significantly predicts resilience $(\beta=0.14, t=3.22, p<0.05)$. Because all the criteria needed to predict mediation were met, a mediation analysis was carried out by using bootstrapping with bias-corrected confidence estimates (Hayes, 2013). A 95\% confidence interval (CI) of the indirect effects was obtained by using 5000 bootstrap resamples. An evaluation of the direct and indirect effects confirmed that mindfulness mediated the relationship between positive affect and resilience $(\beta=0.09 ; \mathrm{CI}=0.030-0.17)$. The results indicated that the positive affect-resilience relationship $(\beta=0.79, t=8.18, p<0.001)$ remained significant when mindfulness was controlled, suggesting that a partial mediation effect is present. The results are shown in Figure 1.

\section{Discussion}

Results of the multiple regression analysis confirmed hypothesis 1, i.e. positive affect is a significant predictor of resilience. These findings are consistent with Cohn, Fredrickson, Brown, Mikels, and Conway (2009) who reported a positive relationship between positive affect and resilience $(r=0.454 ; p \leq 0.001)$. Additionally, Gloria, Faulk, and Steinhardt (2013) reported that in their study, positive affect was largely correlated with resilience $(r=0.65$, $p \leq 0.001$ ). The authors also reported that positive affect explained $37 \%$ of the variance in resilience, after controlling

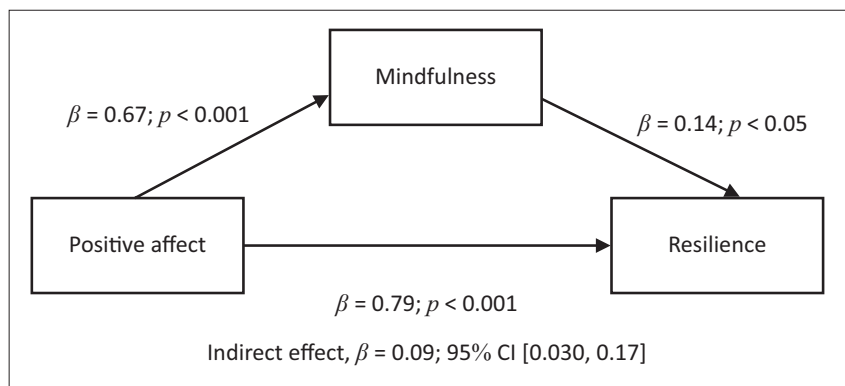

FIGURE 1: The mediating effect of mindfulness on the relationship between positive affect and resilience.

TABLE 3: Multiple regression with resilience being the dependent variable, and positive affect and mindfulness being the independent variables.

\begin{tabular}{|c|c|c|c|c|c|c|c|c|}
\hline \multirow[t]{2}{*}{ Model } & \multicolumn{2}{|c|}{ Unstandardised coefficients } & \multirow{2}{*}{$\begin{array}{c}\text { Standardised coefficients } \\
\boldsymbol{\beta} \\
\end{array}$} & \multirow[t]{2}{*}{$t$} & \multirow[t]{2}{*}{$p$} & \multirow[t]{2}{*}{$F$} & \multirow[t]{2}{*}{$R^{2}$} & \multirow[t]{2}{*}{$\Delta R^{2}$} \\
\hline & $\beta$ & SE & & & & & & \\
\hline (Constant) & 42.944 & 4.081 & - & 10.524 & 0.000 & 50.59 & 0.286 & 0.281 \\
\hline Positive affect & 0.791 & 0.097 & 0.455 & 8.189 & $0.000^{*}$ & - & - & - \\
\hline Mindfulness & 0.135 & 0.042 & 0.179 & 3.227 & $0.001 *$ & - & - & - \\
\hline
\end{tabular}

$t$, test; $p$, probability value; $F$, overall significance; $R^{2}$, percentage variance explained.

$\Delta R^{2}$, change in percentage variance explained; $\beta$, regression coefficient; $\mathrm{SE}$, standard error.

$*, p \leq 0.001$. 
for the effects of the demographic variables and work stress in their sample. In an attempt to investigate the relationship between positive affect and resilience, Fredrickson, Tugade, Waugh, and Larkin (2003) reported convincing evidence that indicates positive affect gives rise to emotions such as interest, happiness, hope and confidence, which was reported to share moderate to strong relationships with resilience ranging from $r=0.40$ to $r=0.52$. Kay (2016) asserts that following from the broaden-and-build theory, individuals who experience frequent positive emotions as a result of positive affect become more satisfied not simply because they are enjoying themselves, but because they have built resources that help deal with a wide range of life's challenges. Kay (2016) concludes that in the face of a plethora of evidence supporting the direct effect of positive affect on resilience, it is safe to conclude that maintaining a positive affect in the wake of an adverse event is necessary for promoting resilience. Fredrickson et al. (2003) state that greater emphasis must be placed on positive affect experienced by resilient individuals and how it works to broaden attention and thinking as this cognitive broadening in turn enables a more effective coping that buffers against adversity and fuels thriving.

Based on the results of the multiple regression analysis, hypothesis 2, i.e. mindfulness is a significant predictor of resilience, is supported. Findings of the current study are consistent with those of Keye and Pidgeon (2013) who reported that mindfulness shared a strong positive relationship with resilience $(r=0.67, p=0.000)$ and that mindfulness explained $44 \%$ of the variance in the levels of resilience. Thompson, Arnkoff, and Glass (2011) state that the relationship between mindfulness and resilience can be explained by focussing on the manner in which mindfulness operates to broaden reaction responses. Thompson et al. (2011) suggest that a mindful and accepting orientation towards experiences helps prevent ruminative and depressive thinking, thereby promoting psychological resilience following a setback. Bajaj and Pande (2016) support this argument by stating that mindful people demonstrate the potential to foster resilience as they are better able to respond to difficult situations without reacting in automatic and nonadaptive ways. The thoughtful reaction to situations allows them to be open to new perceptual categories, develop creative ways of responding and become better at coping with difficult thoughts and emotions without becoming overwhelmed or shutting down. This increases the likelihood of a resilient outcome.

Results of the mediation analysis indicate that mindfulness partially mediated the relationship between positive affect and resilience. These results support hypothesis 3, i.e. there is an indirect relationship between positive affect and resilience through mindfulness. Although these results are significant, the indirect effect of positive affect on resilience through mindfulness is minimal. This suggests that although mindfulness does influence the relationship between positive affect and resilience, the direct effect of positive affect on resilience still appears to be stronger than the effect of positive affect on resilience through mindfulness. These findings indicate the women leaders in the current sample rely more on positive affect to increase resilience than on mindfulness. This finding is expected because previous studies (Cohn et al., 2009; Gloria et al., 2013) have found that positive affect predicts resilience by a large margin, explaining between $30 \%$ and $40 \%$ of the variance in resilience. A plausible explanation as to why the direct effect of positive affect on resilience is stronger than that of positive affect on resilience through mindfulness could lie in the concept of psychological capital. Psychological capital is defined as a personal resource that consists of hope, optimism, self-efficacy and resilience (Seligman \& Csikszentmihalyi, 2000). According to Fredrickson et al. (2003), hope and optimism are positive emotions, which are a result of maintaining a positive affect. Because hope and optimism like resilience are personal resources falling under psychological capital, it is understandable that they strongly predict resilience as several studies have reported strong correlations between sub-dimensions of psychological capital (Herbert, 2011; Liran \& Miller, 2019; Simons \& Buitendach, 2013). According to Herbert (2011), optimistic people tend to draw heavily on favourable dispositional attitudes and behaviours like internal locus of control and empathy to face life stresses, which allows them to remain resilient. Additionally, Herbert (2011) states that hope can only be experienced when the temptation to despair exists. As a result, individuals high in hope will be able to quickly bounce back (resilience) after temporary setbacks. Thus, through increasing optimism and hope, positive affect will strongly explain the variance in resilience. Therefore, mindfulness could not have possibly added more variance to resilience than that created by positive affect as positive affect emphasises hope and optimism.

\section{Practical implications}

The results of the study suggest that given the strong evidence explaining the predictive role of positive affect on resilience, higher education institutions should consider creating opportunities to increase positive affect and mindfulness for women leaders. Higher education institutions should ensure that an organisational climate that facilitates the development of positive affect and mindfulness is created. Additionally, higher education institutions can ensure that current leadership support programmes that target women leaders provide opportunities for cultivating positive affect and mindfulness.

According to Wall, Russell, and Moore (2017), there has been considerable research that suggests organisations can stimulate the growth of positive affect by adapting appreciative inquiry interventions or methods as part of a work-based learning project. Appreciative enquiry emphasises a strength-based approach and highlights elements that are positive in the organisation. By highlighting 
strengths, women leaders will become appreciative for what they have, which nurtures a positive affect. Schein (2010) suggests that organisations should strive to embed drivers of positive affect within the work culture. As a result, attempts made to enhance the positive affect of women leaders should begin with a comprehensive analysis of the prevailing institutional culture.

Results of the current study also suggest that mindfulness can serve as an important internal resource to increase resilient responses. As a result, higher education institutions should consider enhancing levels of mindfulness through mindfulness training. This can be performed through connecting mindfulness to corporate values, supplementing resilience development programmes with mindfulnessbased practice and allowing for mindful moments. Mindful moments can be created through incorporating meditation and exercises to increase focus, which can form part of the institution's wellness programme. With regard to connecting mindfulness to corporate values, institutions should think of linking the benefits of mindfulness to the organisations' stated values. Organisations should attempt to highlight how mindfulness practice can be used to assist in furthering the institutional strategy.

\section{Limitations and recommendations}

This study contributed towards research that aims to build resilience amongst women leaders, with the results suggesting that if positive affect and mindfulness are enhanced amongst aspiring women leaders it is likely that their levels of resilience will also be enhanced. The use of a cross-sectional survey design implies that no conclusion can be made regarding whether positive affect and mindfulness predict resilience over a longer period of time. As a result, it is difficult to predict whether women leaders in the present study make use of positive affect and mindfulness over a period of time or just for the period during which the questionnaires were responded to. Future studies are recommended to adopt a longitudinal design to determine the causal order of constructs and ascertain whether positive affect and mindfulness are consistently used by women leaders.

\section{Conclusion}

Although studies have focused on the role of resilience in general leadership development, the current study contributes to the limited literature that explores internal resources women leaders use to increase resilience. Given the unique barriers women face in the higher education sector, there is a definite need for studies to explore factors that serve to enhance women leaders' resilience.

The results of the current study provided useful insights into the role of psychological strengths such as positive affect and mindfulness in increasing resilience. Institutions can assist in increasing positive affect through the creation of positive experiences for women leaders such as opportunities for coaching and mentoring. Additionally, institutions can encourage the practice of mindfulness through creating opportunities for meditation and exercises to increase attention and awareness to present experiences.

\section{Acknowledgements}

The data were extracted from a larger project, that is, Daphne Pillay's PhD thesis. The research supervisors Prof. E. Van Zyl and Prof. P. Nel (both from the University of the Free State) must be acknowledged for their role in the bigger project. However, for this article all the literature review, statistical analysis and writing were performed solely by D. Pillay.

\section{Competing interests}

The author declares that no competing interest exists.

\section{Author's contributions}

The author declares that she is the sole author of this research article.

\section{Funding information}

This research received no specific grant from any funding agency in the public, commercial or not-for profit sector.

\section{Data availability statement}

Data sharing in not applicable to this article as no new data were created or analysed in this study.

\section{Disclaimer}

The views and opinions expressed in this article are those of the author and do not necessarily reflect the official policy or position of any affiliated agency of the author.

\section{References}

Adams, J., Brewer, S., Chapman-Clarke, M., Chaskalson, M., Furr, M., ...Watt, T. (2016) Building the case for mindfulness in the workplace. London: The Mindfulness Initiative.

Arewasikporn, A., Turner, A., Alschuler, K., Ehde, D., \& Jensen, M. (2018). Positive affect, resilience, and function in adults with multiple sclerosis and chronic pain. The Journal of Pain, 19(3), S32. https://doi.org/10.1016/j.jpain.2017. 12.080

Aydemir, S. (2018). Analysis of psychological resilience in employees in terms of positive and negative affect. Afyon Kocatepe Üniversitesi Sosyal Bilimler Dergisi, 20(3), 69-94. https://doi.org/10.32709/akusosbil.447382

Bajaj, B., \& Pande, N. (2016). Mediating role of resilience in the impact of mindfulness on life satisfaction and affect as indices of subjective well-being. Personality and Individual Differences, 93(1), 63-67. https://doi.org/10.1016/j. paid.2015.09.005

Bartz, A.L. (2017). Using mindfulness to self-regulate in the upper elementary classroom. Unpublished master's thesis. St Paul, MN: St Catherine University.

Bishop, S.R., Lau, M., Shapiro, S., Carlson, L., Anderson, N.D., Carmody, J., \& Devins, G. (2004). Mindfulness: A proposed operational definition. Clinical Psychology: Science and Practice, 11(3), 230-241. https://doi.org/10.1093/ clipsy.bph077

Brosch, T., Scherer, K.R., Grandjean, D.M., \& Sander, D. (2013). The impact of emotion on perception, attention, memory, and decision-making. Swiss Medical Weekly, 143(1), w13786. https://doi.org/10.4414/smw.2013.13786 
Brown, K.W., \& Ryan, R.M. (2003). The benefits of being present: Mindfulness and its role in psychological well-being. Journal of Personality and Social Psychology, 84(4), 822. https://doi.org/10.1037/0022-3514.84.4.822

Chmitorz, A., Kunzler, A., Helmreich, I., Tüscher, O., Kalisch, R., Kubiak, T., ... Lieb, K. (2018). Intervention studies to foster resilience - A systematic review and proposal for a resilience framework in future intervention studies. Clinical Psychology Review, 59(1), 78-100. https://doi.org/10.1016/j.cpr.2017.11.002

Cohn, M.A., Fredrickson, B.L., Brown, S.L., Mikels, J.A., \& Conway, A.M. (2009). Happiness unpacked: Positive emotions increase life satisfaction by building resilience. Emotion, 9(3), 361. https://doi.org/10.1037/a0015952

Collins, K.R., Stritzke, W.G., Page, A.C., Brown, J.D., \& Wylde, T.J. (2018). Mind full of life: Does mindfulness confer resilience to suicide by increasing zest for life? Journal of Affective Disorders, 226, 100-107. https://doi.org/10.1016/j. jad.2017.09.043

Dane, E. (2011). Paying attention to mindfulness and its effects on task performance in the workplace. Journal of Management, 37(4), 997-1018. https://doi. org $/ 10.1177 / 0149206310367948$

Dannels, L.P., \& Masters, J.D. (2020). Getting executives to SIT: Building leaders' resilience and stress tolerance for enhanced organizational effectiveness. Development and Learning in Organizations: An International Journal, 17(1), 1-4. https://doi.org/10.1108/DLO-05-2019-0122

Fergus, S., \& Zimmerman, M.A. (2005). Adolescent resilience: A framework for understanding healthy development in the face of risk. Annual Review Public Health, 26, 399-419. https://doi.org/10.1146/annurev.publhealth.26.021304. 144357

Foerster, C., \& Duchek, S. (2018). Leaders' resilience - A systematic literature review and future research agenda. In Proceedings of the 78th Annual Meeting of the Academy of Management, AOM 2018. Chicago, IL: Academy of Management.

Folkman, S., \& Moskowitz, J.T. (2000). Positive affect and the other side of coping. American Psychologist, 55(6), 647. https://doi.org/10.1037//0003-066x.55.6.647

Fredrickson, B.L. (2001). The role of positive emotions in positive psychology: The broaden-and-build theory of positive emotions. American Psychologist, 56(3) 218. https://doi.org/10.1037/0003-066X.56.3.218

Fredrickson, B.L. (2004). The broaden-and-build theory of positive emotions. Philosophical Transactions of the Royal Society: Biological Sciences, 359(1449), 1367. https://doi.org/10.1098/rstb.2004.1512

Fredrickson, B.L., Tugade, M.M., Waugh, C.E., \& Larkin, G.R. (2003). What good are positive emotions in crisis? A prospective study of resilience and emotions following the terrorist attacks on the United States on September 11th, 2001 Journal of Personality and Social Psychology, 84(2), 365. https://doi. org/10.1037//0022-3514.84.2.365

Gloria, C.T., Faulk, K.E., \& Steinhardt, M.A. (2013). Positive affectivity predicts successful and unsuccessful adaptation to stress. Motivation and Emotion, 37(1) 185-193. https://doi.org/10.1007/s11031-012-9291-8

Grover, S.L., Teo, S.T., Pick, D., Roche, M., \& Newton, C.J. (2018). Psychological capital as a personal resource in the JD-R model. Personnel Review, 47(4), 968-984. https://doi.org/10.1108/PR-08-2016-0213

Halliday, A.J. (2018). Mindful resilience: Investigating mindfulness and resilience in relation to a broad range of adversity. Unpublished doctoral thesis. University of Western Ontario, Canada.

Hamby, S., Banyard, V., \& Grych, J. (2016). Strengths, narrative, and resilience: Restorying resilience research. Psychology of Violence, 6(1), 1. https://doi. org/10.1037/vio0000027

Hayes, A.F. (2013). Introduction to mediation, moderation, and conditional process analysis: A regression-based approach. London: Guilford Press.

Herbert, M. (2011). An exploration of the relationships between psychological capital (hope, optimism, self-efficacy, resilience), occupational stress, burnout and employee engagement. Unpublished Doctoral dissertation. Stellenbosch: Stellenbosch University.

Hoddinott, S.N., \& Bass, M.J. (1986). The Dillman total design survey method. Canadian Family Physician, 32, 2366.

Howard, S., \& Johnson, B. (2002). Participation and involvement: Resilience promoting factors for young adolescents. In M. Gollop \& J. McCormack (Eds.), Children and young people's environments (pp. 113-127). Dunedin: Children's Issues Centre.

Howe-Walsh, L., \& Turnbull, S. (2016). Barriers to women leaders in academia: Tales from science and technology. Studies in Higher Education, 41(3), 415-428. https://doi.org/10.1080/03075079.2014.929102

Huysamen, G.K. (2001). Marking standards and the differential predictability of the first-year university performance of different demographic groups. South African Journal of Higher Education, 15(1), 129-137. https://doi.org/10.4314/sajhe. v15i1.25388

Kay, S.A. (2016). Emotion regulation and resilience: Overlooked connections. Industrial and Organizational Psychology, 9(2), 411-415. https://doi.org/10.1017/iop 2016.31

Keng, S.L., Smoski, M.J., \& Robins, C.J. (2011). Effects of mindfulness on psychological health: A review of empirical studies. Clinical Psychology Review, 31(6), 1041-1056. https://doi.org/10.1016/j.cpr.2011.04.006

Keye, M.D., \& Pidgeon, A.M. (2013). Investigation of the relationship between resilience, mindfulness, and academic self-efficacy. Open Journal of Social Sciences, 1(06), 1-4. 1https://doi.org/10.4236/jss.2013.16001.

Kinman, G., \& Grant, L. (2017). Building resilience in early-career social workers: Evaluating a multi-modal intervention. British Journal of Social Work, 47(7), 1979-1998. https://doi.org/10.1093/bjsw/bcw164
Kong, L., Fang, M., Ma, T., Li, G., Yang, F., Meng, Q., ... \& Li, P. (2018). Positive affect mediates the relationships between resilience, social support and posttraumatic growth of women with infertility. Psychology, Health \& Medicine, 23(6), 707-716. https://doi.org/10.1080/13548506.2018.1447679

Kret, M.E., \& Bocanegra, B.R. (2016). Adaptive hot cognition: How emotion drives information processing and cognition steers affective processing. Frontiers in Psychology, 7, 1920. https://doi.org/10.3389/fpsyg.2016.01920

Ledesma, J. (2014). Conceptual frameworks and research models on resilience in leadership. Sage Open, 4(3), 4. https://doi.org/10.1177/2158244014545464

Lee, T.Y., Cheung, C.K., \& Kwong, W.M. (2012). Resilience as a positive youth development construct: A conceptual review. The Scientific World Journal, 2012, 1-9. Article ID 390450. https://doi.org/10.1100/2012/390450

Liran, B.H., \& Miller, P. (2019). The role of psychological capital in academic adjustment among university students. Journal of Happiness Studies, 20(1), 51-65. https:// doi.org/10.1007/s10902-017-9933-3

Manomenidis, G., Panagopoulou, E., \& Montgomery, A. (2019). Resilience in nursing The role of internal and external factors. Journal of Nursing Management, 27(1) 172-178. https://doi.org/10.1111/jonm.12662

Martínez-Martí, M.L., \& Ruch, W. (2017). Character strengths predict resilience over and above positive affect, self-efficacy, optimism, social support, self-esteem, and life satisfaction. The Journal of Positive Psychology, 12(2), 110-119. https:// doi.org/10.1080/17439760.2016.1163403

Masten, A.S. (2011). Resilience in children threatened by extreme adversity: Frameworks for research, practice, and translational synergy. Development and Psychopathology, 23(2), 493-506. https://doi.org/10.1017/ S0954579411000198

Mould, J. (2014). A programme to promote internal resiliency amongst female adolescents a cognitive behavioural approach. Unpublished doctoral thesis. University of the Free State, South Africa.

Nulty, D.D. (2008). The adequacy of response rates to online and paper surveys: What can be done? Assessment \& Evaluation in Higher Education, 33(3), 301-314. https://doi.org/10.1080/02602930701293231

Ong, A.D., Kim, S., Young, S., \& Steptoe, A. (2017). Positive affect and sleep: A systematic review. Sleep Medicine Reviews, 35, 21-32. https://doi.org/10.1016/j. smrv.2016.07.006

Oshri, A. (2019). Resilience in developmental science: A case for hormesis. Retrieved from https://doi.org/10.31234/osf.io/dwsjg

Petrosyants, V.R. (2017). Features of resilience in psychology students depending on the year of study. Psychological Science and Education, 22(6), 109-117. https:// doi.org/10.17759/pse.2017220610

Richardson, G.E., Neiger, B.L., Jensen, S., \& Kumpfer, K. (1990). The resiliency model. Health Education, 2(6), 33-39. https://doi.org/10.1080/00970050.1990. 10614589

Rupprecht, S., Falke, P., Kohls, N., Tamdjidi, C., Wittmann, M., \& Kersemaekers, W. (2019). Mindful leader development: How leaders experience the effects of mindfulness training on leader capabilities. Frontiers in Psychology, 10, 1081. hittps://doi.org/10.3389/fpsyg.2019.01081

Ryan, R.M., \& Deci, E.L. (2000). Intrinsic and extrinsic motivations: Classic definitions and new directions. Contemporary Educational Psychology, 25(1), 54-67. https:// doi.org/10.1006/ceps.1999.1020

Sanaghan, P. (2016). Building leadership resilience in higher education. Academic Impressions. Retrieved from https://www.academicimpressions.com/sites/ default/files/0116-leadership-resilience-md.pdf

Schein, E.H. (2010). Organizational culture and leadership (Vol. 2). Hoboken, NJ: John Wiley \& Sons

Seligman, M.E., \& Csikszentmihalyi, M. (2000). Positive psychology: An introduction American Psychological Association, 55(1), 5. https://doi.org/10.1037/0003066X.55.1.5

Simons, J.C., \& Buitendach, J.H. (2013). Psychological capital, work engagement and organisational commitment amongst call centre employees in South Africa. SA Journal of Industrial Psychology, 39(2), 1-12. https://doi.org/10.4102/sajip. v39i2.1071

Sommer, S.A., Howell, J.M., \& Hadley, C.N. (2016). Keeping positive and building strength: The role of affect and team leadership in developing resilience during an organizational crisis. Group \& Organization Management, 41(2), 172-202. https://doi.org/10.1177/1059601115578027

Thompson, R.W., Arnkoff, D.B., \& Glass, C.R. (2011). Conceptualizing mindfulness and acceptance as components of psychological resilience to trauma. Trauma, Violence, \& Abuse, 12(4), 220-235. https://doi.org/10.1177/ 1524838011416375

Tugade, M.M., \& Fredrickson, B.L. (2004). Resilient individuals use positive emotions to bounce back from negative emotional experiences. Journal of Personality and Social Psychology, 86(2), 320. https://doi.org/10.1037/0022 3514.86.2.320

Tyng, C.M., Amin, H.U., Saad, M.N., \& Malik, A.S. (2017). The influences of emotion on learning and memory. Frontiers in Psychology, 8, 1454. https://doi.org/10.3389/ fpsyg.2017.01454

Ungar, M. (2004). A constructionist discourse on resilience: Multiple contexts, multiple realities among at-risk children and youth. Youth \& Society, 35(3), 341-365. https://doi.org/10.1177/0044118X03257030

Van Cappellen, P., Toth-Gauthier, M., Saroglou, V., \& Fredrickson, B.L. (2016). Religion and well-being: The mediating role of positive emotions. Journal of Happiness Studies, 17(2), 485-505. https://doi.org/10.1007/s10902-014-9605-5 
Wagnild, G., \& Young, H. (1993). Development and psychometric evaluation of the resilience scale. Journal of Nursing Measurement, 1(2), 165-178.

Wagnild, G., \& Young, H. (2009). Development and psychometric. Journal of Nursing Measurement, 17(2), 165-178.

Wall, T., Russell, J., \& Moore, N. (2017). Positive emotion in workplace impact: The case of a work-based learning project utilising appreciative inquiry. Journal of Work-Applied Management, 9(2), 129-146. https://doi.org/10.1108/JWAM-072017-0017
Watson, D., Clark, L.A., \& Tellegen, A. (1988). Development and validation of brief measures of positive and negative affect: The PANAS scales. Journal of
Personality and Social Psychology, 54(6), 1063. https://doi.org/10.1037//00223514.54.6.1063

Weatherspoon-Robinson, S. (2013). African American female leaders: Resilience and success. Unpublished doctoral dissertation. Malibu, CA: Pepperdine University.

Werner, E.E., \& Smith, R.S. (1992). Overcoming the odds: High risk children from birth to adulthood. Ithaca, NY: Cornell University Press. 